\title{
High energy effects on $D$-brane and black hole emission rates
}

\author{
Saurya Das, ${ }^{*}$ Arundhati Dasgupta, ${ }^{\dagger}$ and Tapobrata Sarkar ${ }^{\ddagger}$ \\ The Institute of Mathematical Sciences, CIT Campus, Chennai (Madras) 600 113, India
}

(Received 18 February 1997)

\begin{abstract}
We study the emission of scalar particles from a class of near-extremal five-dimensional black holes and the corresponding $D$-brane configuration at high energies. We show that the distribution functions and the black hole greybody factors are modified in the high energy tail of the Hawking spectrum in such a way that the emission rates exactly match. We extend the results to charged scalar emission and to four dimensions. [S0556-2821(97)02712-4]
\end{abstract}

PACS number(s): 04.70.Dy, 04.62.+v, 11.25.Mj

\section{INTRODUCTION}

Recently, it was shown that a microscopic interpretation can be given to Bekenstein-Hawking entropy of certain stringy black holes. These black holes can be identified with elementary or solitonic string states and the degeneracy of the latter matches with the entropy of the black hole [1-3]. Hawking radiation of scalars has also been understood in terms of their $D$-brane description. The Planck factor in the Hawking spectrum was obtained in $[4,5]$ using the distribution functions of open string states residing on the $D$ branes. Shortly, it was shown in [6] that the rate of $D$ branes decaying into low energy scalars perfectly matched the Hawking spectrum from the corresponding black hole. The black holes considered were solutions of low energy effective action of type-IIB string theory compactified on a five-dimensional torus. Their $D$-brane description consisted of $Q_{5}$ $D$-5-branes wrapped around $T^{5}$ and $Q_{1} D$-1-branes wrapped around $S^{1}$ contained in the $T^{5}$ and a collection of open strings carrying some momentum along $S^{1}$. The situation is equivalent to a single "long", $D$-1-brane wrapped $Q_{1} Q_{5}$ times around the $S^{1}[5,7]$. The left- and right-moving massless open string states on this long brane constitute two noninteracting one-dimensional gases, approximated by canonical ensembles at low energies. A pair of oppositely moving states, each carrying energy $\omega / 2$, can annihilate to form a closed string state, like the graviton in the internal dimensions, of energy $\omega$, which cannot reside on the $D$ brane and is emitted as a scalar particle. The exact decay rate can be calculated from the Dirac-Born-Infeld action, which to leading order is given by

$$
\Gamma_{D}=g_{\text {eff }} \omega \rho\left(\frac{\omega}{2 T_{L}}\right) \rho\left(\frac{\omega}{2 T_{R}}\right) \frac{d^{4} k}{(2 \pi)^{4}},
$$

where $\rho\left(\omega / 2 T_{L, R}\right)=1 /\left[\exp \left(\omega / 2 T_{L, R}\right)-1\right]$ and $g_{\text {eff }}$ is related to the parameters of the corresponding black hole. $T_{L}$ and $T_{R}$ are the effective temperatures of the left- and rightmoving canonical ensembles. In the limit $T_{L} \gg T_{R}$, it was shown that [6]

\footnotetext{
*Electronic address: saurya@imsc.ernet.in

†Electronic address: dasgupta@imsc.ernet.in

¥Electronic address: sarkar@imsc.ernet.in
}

$$
\Gamma_{D}=A_{H} \rho\left(\frac{\omega}{T_{H}}\right) \frac{d^{4} k}{(2 \pi)^{4}},
$$

where $T_{H}$ is the Hawking temperature of the black hole [6]. On the other hand, the Hawking spectrum from the black hole is given by

$$
\Gamma_{H}=\sigma_{\mathrm{abs}} \rho\left(\frac{\omega}{T_{H}}\right) \frac{d^{4} k}{(2 \pi)^{4}},
$$

where $\sigma_{\mathrm{abs}}$ is the greybody factor for the black hole, which for low energy emissions is just the area of the event horizon $A_{H}$. Substituting, we find that the rates (2) and (3) match exactly.

In [10], the restriction $T_{L} \gg T_{R}$ was dropped, while still remaining in the near-extremal region, and it was shown in general that, for $T_{L} \sim T_{R}$,

$$
\sigma_{\mathrm{abs}}=g_{\mathrm{eff}} \omega \frac{e^{\omega / T_{H}}-1}{\left(e^{\omega / 2 T_{L}}-1\right)\left(e^{\omega / 2 T_{R}}-1\right)},
$$

with the Hawking temperature given by

$$
\frac{2}{T_{H}}=\frac{1}{T_{L}}+\frac{1}{T_{R}} .
$$

Once again, it is seen from Eqs. (1) and (3), that the $D$-brane and the black hole decay rates match.

In the above analyses, it was strictly assumed that the energy of the emitted scalars was vanishingly small. Recently, the high energy tail of the emission spectrum for $D$-branes as well as black holes was probed in [8] although confined to the $T_{L} \gg T_{R}$ regime. The energy $\omega$ was chosen such that $T_{R}, T_{H} \ll \omega \ll T_{L}$. In this regime, the right-moving open strings were treated as a microcanonical ensemble and the corresponding distribution function was modified to

$$
\rho\left(\frac{\omega}{2 T_{R}}\right) \approx \exp \left[S_{R}\left(N_{R}^{\prime}-m\right)-S_{R}\left(N_{R}^{\prime}\right)\right]=\exp \left(-\Delta S_{R}\right),
$$

where $\Delta S_{R}$ is the change in the right-moving entropy on removal of a boson at level $m$ with energy $\omega / 2$. Here $N_{R}^{\prime}$ and $N_{L}^{\prime}$ are the left- and right-moving momenta on the long $D$-1-brane, respectively (the actual momenta on the one 
brane is given in terms the quantum number $\left.N_{L, R}=N_{L, R}^{\prime} / Q_{1} Q_{5}\right)$. Now, the black hole entropy is given by $[6,8]$

$$
S_{\mathrm{BH}}=2 \pi\left(\sqrt{N_{L}^{\prime}}+\sqrt{N_{R}^{\prime}}\right) .
$$

In the limit $T_{L} \gg T_{R}$, since $N_{L}^{\prime} \gg N_{R}^{\prime}$, we get from Eq. (7), $\Delta S_{\mathrm{BH}} \approx \Delta S_{R}$. Thus,

$$
\rho\left(\frac{\omega}{2 T_{R}}\right) \approx \exp \left[S_{\mathrm{BH}}(M-\omega)-S_{\mathrm{BH}}(M)\right]=\exp \left(-\Delta S_{\mathrm{BH}}\right),
$$

where $\Delta S_{\mathrm{BH}}$ is the change in the entropy of the black hole of initial mass $M$ after it emits the Hawking particle of energy $\omega$.

On the black hole side, this change in the distribution function has been attributed to the back reaction effects which become important at high energies. In $[14,8]$ this was studied by modeling the outgoing particle as a spherical shell and quantizing it. In the WKB approximation, the Hawking factor $\rho\left(\omega / T_{H}\right)$ turned out to be precisely the right-hand side of Eq. (8). The left distribution function and the greybody factor remains unchanged and thus, once again the $D$-brane and black hole emission rates are found to match.

In this paper, we will relax the condition $T_{L} \gg T_{R}$ and investigate the range $\omega \gg T_{L, R, H}$. The gas of open strings is treated as a microcanonical ensemble in both the left and the right sectors. We show that the greybody factor gets significantly modified in the high energy tail of the spectrum. With these, we find that the emission rates once again match. Finally, we generalize the results for charged scalar emission and to four dimensions.

\section{D-BRANE EMISSION SPECTRUM AT HIGH ENERGIES}

Consider a one-dimensional gas of massless open strings in a box of length $L$. The total momentum $P$ of the gas is given in terms of the quantum number $N^{\prime}$ by $P=2 \pi N^{\prime} / L$ and the energy of a colliding string by

$$
\omega / 2=2 \pi m / L
$$

For low energy excitations, such that $m \ll \sqrt{N^{\prime}}$, the gas is well approximated by a canonical ensemble, and the distribution function is of the Bose-Einstein form. However, for higher energies, when

$$
\sqrt{N^{\prime}} \ll m \ll N^{\prime},
$$

which amounts to the excitation energy being much greater than the corresponding temperature, the canonical description is inadequate, and the gas should be described by a microcanonical ensemble. Since we are interested in the regime $T_{L} \sim T_{R}$ and $\omega$ exceeds these temperatures, the microcanonical distribution functions should be invoked in the right as well as the left sectors, which is given by [8]

$$
\rho\left(\frac{\omega}{2 T_{L, R}}\right)=\exp \left[-2 \pi\left(\sqrt{N_{L, R}^{\prime}}-\sqrt{N_{L, R}^{\prime}-m}\right)\right] .
$$

From Eqs. (7) and (9), we write

$$
\begin{aligned}
\rho\left(\frac{\omega}{2 T_{L}}\right) \rho\left(\frac{\omega}{2 T_{R}}\right)= & \exp \left\{-2 \pi\left[\sqrt{N_{L}^{\prime}}+\sqrt{N_{R}^{\prime}}\right.\right. \\
& \left.-\sqrt{N_{L}^{\prime}-L\left(\frac{\omega}{4 \pi}\right)}-\sqrt{\left.N_{R}^{\prime}-L\left(\frac{\omega}{4 \pi}\right)\right]}\right\} \\
= & \exp \left(-\Delta S_{L}-\Delta S_{R}\right)=\exp \left(-\Delta S_{\mathrm{BH}}\right) .
\end{aligned}
$$

Thus, Eq. (1) can be written as

$$
\Gamma_{D}=g_{\text {eff }} \omega \exp \left(-\Delta S_{\mathrm{BH}}\right) \frac{d^{4} k}{(2 \pi)^{4}} .
$$

In the black hole side, the Hawking factor becomes, on inclusion of back reaction [8],

$$
\rho\left(\frac{\omega}{T_{H}}\right) \approx \exp \left[S_{\mathrm{BH}}(M-\omega)-S_{\mathrm{BH}}(M)\right]=\exp \left(-\Delta S_{\mathrm{BH}}\right),
$$

which implies

$$
\Gamma_{H}=\sigma_{\mathrm{abs}} \exp \left(-\Delta S_{\mathrm{BH}}\right) \frac{d^{4} k}{(2 \pi)^{4}} .
$$

In the next section, we will calculate $\sigma_{\text {abs }}$ and compare the $D$-brane and black hole emission rates. Note that, in [8] the left sector did not contribute to $\Delta S_{\mathrm{BH}}$ and the relation between the distribution functions was

$$
\rho\left(\frac{\omega}{2 T_{R}}\right)=\rho\left(\frac{\omega}{T_{H}}\right) .
$$

Here, on the other hand, both the sectors become equally important and contribute to the Hawking factor.

\section{BLACK HOLE GREYBODY FACTORS AT HIGH ENERGIES}

In this section, we calculate the greybody factors for the five-dimensional black hole under consideration for quanta of high energies. We follow the methods of [9-11]. This is appropriate in the energy regime where back reaction becomes important. We solve the Klein-Gordon equation in the background of the metric given by [12]

$$
\begin{aligned}
d s^{2}= & \frac{1}{\left(f_{1} f_{2} f_{3}\right)^{2 / 3}}\left[-d t^{2}\left(1-\frac{r_{0}^{2}}{r^{2}}\right)\right]+\left(f_{1} f_{2} f_{3}\right)^{1 / 3} \\
& \times\left[\left(1-\frac{r_{0}^{2}}{r^{2}}\right)^{-1} d r^{2}+r^{2} d \Omega_{3}^{2}\right],
\end{aligned}
$$

where

$$
f=\left(1+\frac{r_{n}^{2}}{r^{2}}\right)\left(1+\frac{r_{1}^{2}}{r^{2}}\right)\left(1+\frac{r_{5}^{2}}{r^{2}}\right)
$$

and

$$
h=1-\frac{r_{0}^{2}}{r^{2}}
$$


The parameters $r_{1}, r_{5}$, and $r_{n}$ can be expressed in terms of the two charges $Q_{1}, Q_{5}$, and the momentum $n$ along the $D$-1-brane as

$$
\begin{gathered}
r_{1}^{2}=\frac{g Q_{1}}{V}, \quad r_{5}^{2}=g Q_{5}, \\
r_{0}^{2} \frac{\sinh 2 \sigma}{2}=\frac{g^{2} n}{R^{2} V}, \quad r_{n}^{2}=r_{0}^{2} \sinh ^{2} \sigma,
\end{gathered}
$$

where $\sigma$ is a boost parameter. The radial part of the KleinGordon equation for a scalar field $\phi$ corresponding to the $s$-wave state and propagating in the background of the above metric is given by

$$
\frac{h}{r^{3}} \frac{d}{d r}\left(h r^{3} \frac{d R}{d r}\right)+\omega^{2} f R=0
$$

In our calculations, we relax the low energy condition $\omega r_{5}, \omega r_{1} \ll 1$, originally imposed in [10] and solve the above equation by treating the new $\omega$-dependent terms that enter due to this relaxation, as a perturbation over the terms originally present. The following analysis is valid so long as $\omega r_{1}, \omega r_{5}<1$, although it need not be vanishingly small. Towards the end of this section, we show that this is the relevant range for comparing with the $D$-brane results. Equation (18) is solved by dividing space into two regions, the near and far zones, and then matching the solutions at some intermediate region. We assume the following relation between the various parameters

$$
r_{0}, \quad r_{n} \ll r_{m}<r_{1}, \quad r_{5},
$$

where the near and far solutions are matched at $r=r_{m}$. In the far region, we get, from Eq. (18),

$$
\frac{d^{2} \psi}{d r^{2}}+\left[\omega^{2}+\frac{-3 / 4+\omega^{2}\left(r_{1}^{2}+r_{5}^{2}\right)}{r^{2}}\right] \psi=0
$$

where we have substituted $R=\psi r^{-3 / 2}$ and the restrictions given in Eq. (19). The term $\omega^{2}\left(r_{1}^{2}+r_{5}^{2}\right)$ was absent in [10] because of the low energy condition. Defining $\rho=\omega r$, we obtain

$$
\frac{d^{2} \psi}{d \rho^{2}}-\left[-1+\frac{3 / 4-\omega^{2}\left(r_{1}^{2}+r_{5}^{2}\right)}{\rho^{2}}\right] \psi=0,
$$

which has the solution

$$
\psi=\sqrt{\frac{\pi}{2} \rho}\left[\alpha J_{1-\epsilon}(\rho)+\beta N_{1-\epsilon}(\rho)\right] .
$$

where $\epsilon \equiv \omega^{2}\left(r_{1}^{2}+r_{5}^{2}\right) / 2$. Now, in the matching region, we use the small $\rho$ expansion for the Bessel functions, and finally obtain, for the solution,

$$
\begin{aligned}
R= & \sqrt{\frac{\pi}{2} \omega^{3 / 2}\left\{\frac{\alpha(\rho / 2)^{-\epsilon}}{2 \Gamma(2-\epsilon)}+\frac{\beta}{2}\left[\left(\frac{\rho}{2}\right)^{-\epsilon} \cot \pi(1-\epsilon)\right.\right.} \\
& \left.\left.-\frac{\epsilon}{\sin \pi(1-\epsilon)}\left(\frac{\rho}{2}\right)^{-2+\epsilon}\right]\right\} .
\end{aligned}
$$

On the other hand, the asymptotic expansions of the Bessel functions yield the solutions

$$
\begin{aligned}
& J_{1-\epsilon}(\rho)=\sqrt{\frac{2}{\pi \rho}} \cos \left(\rho-\frac{3 \pi}{4}+\frac{\pi \epsilon}{2}\right), \\
& N_{1-\epsilon}(\rho)=\sqrt{\frac{2}{\pi \rho}} \sin \left(\rho-\frac{3 \pi}{4}+\frac{\pi \epsilon}{2}\right),
\end{aligned}
$$

which are used to compute the incoming flux at infinity, given by

$$
\Phi_{\text {in }}=-\frac{\omega}{4}|\alpha|^{2}
$$

In this computation, we have dropped a $\beta$ dependent piece. From Eq. (23), it is clear that the term multiplying $\beta$ is large for small values of the perturbation parameter. This implies that $\beta / \alpha \ll 1$.

In the near zone, Eq. (18) can be written as

$\frac{h}{r^{3}} \frac{d}{d r}\left(h r^{3} \frac{d R}{d r}\right)+\omega^{2}\left[\frac{\left(r_{n} r_{1} r_{5}\right)^{2}}{r^{6}}+\frac{\left(r_{1} r_{5}\right)^{2}}{r^{4}}+\frac{\left(r_{1}^{2}+r_{5}^{2}\right)}{r^{2}}\right] R=0$.

Defining new variables $v$ and parameters $A, B$ as

$$
v=\frac{r_{0}^{2}}{r^{2}} ; \quad A=\frac{\omega^{2}}{4}\left(\frac{r_{1} r_{5} r_{n}}{r_{0}}\right)^{2} ; B=\frac{\omega^{2}}{4}\left(\frac{r_{1} r_{5}}{r_{0}}\right)^{2}
$$

Eq. (26) becomes

$$
(1-v) \frac{d}{d v}\left((1-v) \frac{d R}{d v}\right)+\left[A+\frac{B}{v}+\frac{\epsilon}{2 v^{2}}\right] R=0 .
$$

Notice that close to the horizon, $v \rightarrow 1^{-}$. Thus, on writing $v=1-\delta$ and expanding the $1 / v^{2}$ term in square brackets, we obtain the equation for the near region as

$$
(1-v) \frac{d}{d v}\left((1-v) \frac{d R}{d v}\right)+\left[A+\frac{B+\epsilon / 2}{v}+\frac{\epsilon \delta}{2}\right] R=0
$$

Hereafter, we drop the $\epsilon \delta / 2$ term, which is very small. In order to compute the flux of neutral scalars absorbed into the black hole, we need to know the near region solution very close to the horizon. In Eq. (28), if we make the substitution $y=-\ln (1-v)$, we obtain, in this region, a simple harmonic equation for $R$ : namely,

$$
\frac{d^{2} R}{d y^{2}}+\left(A+B+\frac{\epsilon}{2}\right) R=0
$$

And the incoming solution is given by

$$
R_{\mathrm{in}}=K \exp \left(-i \sqrt{A+B+\frac{\epsilon}{2}} \ln (1-v)\right) .
$$

Substituting $z=(1-v)$, and writing an ansatz for the solution as $R=K z^{-i(p+q) / 2} R_{1}$, we obtain 


$$
z(1-z) \frac{d^{2} R_{1}}{d z^{2}}+(1-z)(1-i p-i q) \frac{d R_{1}}{d z}+p q R_{1}=0 .
$$

This is seen to be a hypergeometric equation in $R$, where we have defined $p$ and $q$ by the equations

$$
(p+q)^{2}=4\left(A+B+\frac{\epsilon}{2}\right) ; p q=B+\frac{\epsilon}{2} \text {. }
$$

The above equation has the solution

$$
R_{1}=F(-i p,-i q, 1-i p-i q, z),
$$

where $F$ is the hypergeometric function, and hence the full solution for $R$ is given by

$$
R=K z^{-i(p+q) / 2} F(-i p,-i q, 1-i p-i q, z) .
$$

Next, we express $p$ and $q$ in terms of the black hole parameters. Solving the Eq. (33) yields, for $p$ and $q$,

$$
\begin{gathered}
p=\frac{\omega r_{1} r_{5}}{2 r_{0}} e^{\sigma}+\frac{\omega r_{0}}{4} \frac{\left(r_{1}^{2}+r_{5}^{2}\right)}{r_{1} r_{5}} \frac{1}{\cosh \sigma}, \\
q=\frac{\omega r_{1} r_{5}}{2 r_{0}} e^{-\sigma}+\frac{\omega r_{0}}{4} \frac{\left(r_{1}^{2}+r_{5}^{2}\right)}{r_{1} r_{5}} \frac{1}{\cosh \sigma} .
\end{gathered}
$$

Substituting for $T_{L, R}$, namely,

$$
T_{L, R}=\frac{r_{0}}{2 \pi r_{1} r_{5}} e^{ \pm \sigma}
$$

and using $r_{1} \sim r_{5}$, we get

$$
p=\frac{\omega}{4 \pi T_{R}}+\frac{\omega r_{0}}{2 \cosh \sigma}
$$

and

$$
q=\frac{\omega}{4 \pi T_{L}}+\frac{\omega r_{0}}{2 \cosh \sigma}
$$

In order to proceed to calculate the absorption cross section, let us first match the far and near zone solutions at $r=r_{m}$. Extrapolating the near solution given by Eq. (35) to the region of small $v$ (large $r$ ) yields

$$
R=K\left[\frac{\Gamma(1-i p-i q)}{\Gamma(1-i p) \Gamma(1-i q)}+v_{m}\left(a+b \ln v_{m}\right)\right]
$$

where $a$ and $b$ are constants depending on $p$ and $q$. Next, we expand the right-hand side of Eq. (23) in powers of $\epsilon$, and retaining the lowest order terms in $\epsilon$, we find the matching condition at $r=r_{m}$ :

$$
\sqrt{\frac{\pi}{2}} \omega^{3 / 2}\left[\frac{\alpha}{2}\left[1-\epsilon \ln \left(\rho_{m} / 2\right)\right]\right]=K\left[E+v_{m}\left(a+b \ln v_{m}\right)\right]
$$

where

$$
E=\frac{\Gamma(1-i p-i q)}{\Gamma(1-i p) \Gamma(1-i q)}
$$

and we have imposed the condition $z \simeq 1$. The matching region is chosen such that $\omega r_{m}$ is slightly less than unity. Thus, the second term on the left-hand side of Eq. (40) can be dropped, and we get the relation as in [10]:

$$
\sqrt{\frac{\pi}{2}} \omega^{3 / 2} \frac{\alpha}{2}=K E \text {. }
$$

Now, let us calculate the absorption cross section [6]. The flux into the black hole, from Eq. (31) is given by

$$
\Phi_{\mathrm{abs}}=-r_{0}^{2}(p+q)|K|^{2} .
$$

From Eqs. (25) and (42), we get

$$
\sigma_{\mathrm{abs}}=\frac{4 \pi}{\omega^{3}} \frac{\Phi_{\mathrm{abs}}}{\Phi_{\mathrm{in}}}=\frac{2 \pi^{2} r_{0}^{2}}{\omega}(p+q) \frac{1}{|E|^{2}} .
$$

Using the identity

$$
|\Gamma(1-i x)|^{2}=\frac{\pi x}{\sinh \pi x},
$$

we get

$$
\frac{1}{|E|^{2}}=\frac{2 \pi p q}{p+q} \frac{\exp [2 \pi(p+q)]-1}{[\exp (2 \pi p)-1][\exp (2 \pi q)-1]} .
$$

Now, recalling the expressions for $p$ and $q$, in Eq. (36), we see that in the limit when $\omega / T_{L}, R \gg 1$, we can ignore the factors of unity in the numerator and denominator and finally we are left with the following expression for the absorption cross section:

$$
\sigma_{\mathrm{abs}}=\sigma_{\mathrm{abs}}^{0}+\sigma_{\mathrm{abs}}^{1},
$$

where $\sigma_{\mathrm{abs}}^{0}=\pi^{3} r_{1}^{2} r_{5}^{2} \omega$ and the correction term $\sigma_{\text {abs }}^{1}=4 \pi^{3} \omega r_{0}^{2} r_{1} r_{5} \cosh \sigma$. Thus, we see, following the relation between the various parameters that we have considered,

$$
\frac{\sigma_{\mathrm{abs}}^{1}}{\sigma_{\mathrm{abs}}^{0}} \sim\left(\frac{r_{0}}{r_{1}}\right)^{2} \ll 1
$$

Using the definition $g_{\text {eff }}=\pi^{3} r_{1}^{2} r_{5}^{2}$, we get

$$
\sigma_{\text {abs }}=g_{\text {eff }} \omega .
$$

Now, let us compare the expressions for the black hole and $D$-brane decay rates at the high energy regime that we are considering. Substituting Eq. (46) in Eq. (15), we see that the black hole decay rate becomes

$$
\Gamma_{H}=g_{\text {eff }} \omega \exp \left(-\Delta S_{\mathrm{BH}}\right) \frac{d^{4} k}{(2 \pi)^{4}}
$$

which is just the $D$-brane decay rate (13). It may be noted that this matching cannot be obtained by naively ignoring the unity factors in Eqs. (1), (3), and (4). This is because of the fact that in the regime of high energy particle emission that we are interested in, the Planckian distribution of the Hawk- 
ing particles is no longer valid and we have to instead resort to Eqs. (12) and (14). Hence our result (46) effects a subtle match between the black hole and $D$-brane decay rates at high energies. It can be shown that in the special case $T_{L} \gg T_{R}(\sigma \rightarrow \infty)$, the results of [8] are reproduced.

A word about the range of validity of the above result is in order. As stated earlier, microcanonical corrections become important when the condition (10) holds. Using Eq. (9) and substituting the expression for temperature [6], namely,

$$
T_{L, R}=\sqrt{\frac{8 E_{L, R}}{L \pi f}}
$$

in Eq. (10), we obtain

$$
\frac{\omega}{T_{L, R}} \gg 1
$$

which was the condition under which we had derived Eq. (46). Hence we see that taking microcanonical corrections into consideration naturally enforces the high energy condition (49). In terms of the black hole parameters, this can be written as

$$
\omega r_{5} \gg \frac{r_{0}}{r_{1}}
$$

Also, our perturbative analysis is valid so long as $\omega r_{5}<1$, which is consistent with the condition $m \ll N_{L, R}^{\prime}$ of Eq. (10). Hence, the range of $\omega$ for which our calculations are valid is

$$
\frac{r_{0}}{r_{1}} \ll \omega r_{5}<1
$$

On the other hand, it is clear that for low energies (canonical distribution) $m \ll \sqrt{N^{\prime}}$ implying $\omega r_{5} \ll r_{0} / r_{1}$. Thus, it is sufficient to calculate the greybody factor for $\omega r_{5} \ll 1$, as in [10]. However, in our case, it becomes important to look at $\sigma_{\text {abs }}$ for higher $\omega$, and Eq. (51) exhausts the range over which the $D$-brane distribution functions follow that of microcanonical ensembles.

\section{CHARGED EMISSION RATES INCLUDING BACK REACTION}

The results of the previous sections can be extended to include charged scalar emission. The decay rate for low energy charged scalar emission from $D$ branes, has been obtained in [13]. The emitted massless graviton field with polarization along the compact directions now have a net momentum along the compact $S^{1}$ direction on which the one-brane is wrapped. The decay rate is given by

$$
\Gamma_{D}=g_{\text {eff }} \frac{\left(\omega^{2}-e^{2}\right)}{\omega} \rho\left(\frac{\omega+e}{2 T_{L}}\right) \rho\left(\frac{\omega-e}{2 T_{R}}\right) \frac{d^{4} k}{(2 \pi)^{4}} .
$$

Comparing with Eq. (1), we find that here the energies and the momenta of the left and right modes are shifted by a factor of $\pm e / 2$, respectively. This ensures that there is a net momentum $e$ in the $S^{1}$ direction, while the energy of the outgoing particle is $\omega$. This net momenta along the compact direction gives rise to a Kaluza-Klein charge $e$ for the space- time scalar. A mass is also endowed such that $|e|=m$. When $T_{L} \gg T_{R}$, and $\omega$ is low, the emission rate is

$$
\Gamma_{D}=\frac{A_{H}(\omega-e)}{\omega} \rho\left(\frac{\omega-e}{2 T_{R}}\right) \frac{d^{4} k}{(2 \pi)^{4}} .
$$

For higher energies, however, the decay rate is modified. In the regime $T_{L} \sim T_{R},(\omega \pm e) / 2 T_{L, R} \gg 1$, the density functions are best approximated as a microcanonical distribution in this regime. The expression for the left and right densities are same as that in Eq. (12), however, with energies $(\omega+e) / 2$ and $(\omega-e) / 2$ of the left and right particles, respectively. The product of the left and right density functions combine to give

$$
\rho\left(\frac{\omega+e}{2 T_{L}}\right) \rho\left(\frac{\omega-e}{2 T_{R}}\right)=\exp \left(-\Delta S_{\mathrm{BH}}\right)
$$

where now $\Delta S_{\mathrm{BH}}$ is given by $\Delta S_{\mathrm{BH}}=S(M, Q)$ $-S(M-\omega, Q-e)$. Here $M$ is the Arnowitt-Deser-Misner (ADM) mass of the black hole and $Q$ its Kaluza-Klein charge, proportional to the momentum $N_{L}-N_{R}$. Clearly $\Delta S_{\mathrm{BH}}$ is the change in entropy due to the emission of a particle with energy $\omega$ and charge $e$. Then Eq. (52) can be written as

$$
\Gamma_{D}=g_{\text {eff }} \frac{\left(\omega^{2}-e^{2}\right)}{\omega} \exp \left(-\Delta S_{\mathrm{BH}}\right) \frac{d^{4} k}{(2 \pi)^{4}} .
$$

The microcanonical decay rate thus obtained can be reproduced exactly from field theory, following [8], using the techniques developed in [14]. Charged black holes emit charged particles at a rate given by

$$
\Gamma_{H}=\frac{\sqrt{\omega^{2}-e^{2}}}{\omega} \sigma_{\mathrm{abs}} \rho\left(\frac{\omega-e}{T_{H}}\right) \frac{d^{4} k}{(2 \pi)^{4}} .
$$

The density function is evaluated by computing Bogoliubov coefficients. These relate the wave function at the horizon to the normal components of the wave function at $r \rightarrow \infty$. Due to the infinite boosts associated with the horizon, the wave function $\phi_{h}$ is well approximated by the WKB value

$$
\phi_{h}=\exp (\imath S) .
$$

The action $S$ is calculated along the trajectory of the charged shell which approximates the outgoing charged scalar wave. The Bogoliubov coefficients are, hence,

$$
\begin{gathered}
\alpha_{\omega \omega^{\prime}}=\frac{1}{u(r, e)} \int_{-\infty}^{\infty} e^{\imath \omega t} e^{\imath S} d t, \\
\beta_{\omega \omega^{\prime}}=\frac{1}{v(r,-e)} \int_{-\infty}^{\infty} e^{-\imath \omega t} e^{\imath S} d t,
\end{gathered}
$$

where $u(r, e)$ and $v(r,-e)$ give the radial wave function of the positive energy, positively charged particle and negative energy and negatively charged particle, respectively. Since $\omega$ and the action diverge near the horizon, the saddle point value of the integral dominates. The saddle point value is determined through the equation 


$$
\frac{\partial S}{\partial t} \pm \omega=0 .
$$

By the Hamilton Jacobi equation, the saddle point corresponds to $\mathcal{H}^{\mp e}=\mp \omega, \mathcal{H}$ being the Hamiltonian of the outgoing particle. Since the trajectory of the charged shell is that of a null geodesic in the metric [14]

$$
\begin{aligned}
d s^{2}= & -\left[N_{t}(M+\mathcal{H}, Q+e) d t\right]^{2} \\
& +\left[d r+N_{r}(M+\mathcal{H}, Q+e) d t\right]^{2},
\end{aligned}
$$

we have

$$
\dot{r}=N_{t}(M+\mathcal{H}, Q+e)-N_{r}(M+\mathcal{H}, Q+e) .
$$

Using this and the fact that $\partial S / \partial r=P(P$ is the canonical conjugate momentum), we find

$$
\begin{aligned}
& \left|\alpha_{\omega \omega^{\prime}}\right|=\exp \left(-\operatorname{Im} \int_{r_{+0}}^{r_{f}} P_{+} d r\right), \\
& \left|\beta_{\omega \omega^{\prime}}\right|=\exp \left(-\operatorname{Im} \int_{r_{-0}}^{r_{f}} P_{-} d r\right),
\end{aligned}
$$

where $P_{ \pm}$correspond to the positive energy, positively charged particle and negative energy, negatively charged particle trajectories, respectively. As in the case of [8], the positive energy trajectory gives a real value of the integration, while there is an imaginary contribution from the other. As $r_{0}=R(M-\omega, Q-e)-\epsilon$,

$$
\begin{aligned}
\operatorname{Im} \int_{r_{o}}^{r_{f}} P_{-} d r & =-\pi \int_{0}^{-\omega} \frac{d \mathcal{H}-\Phi d Q}{\kappa(M+\mathcal{H}, Q-e)} \\
& =-\frac{1}{2} \int_{M, Q}^{M-\omega, Q-e} d S_{\mathrm{BH}},
\end{aligned}
$$

where we have used $\dot{r} d P=d \mathcal{H}-\Phi d Q$ near the horizon and $d \mathcal{H}=(\kappa / 2 \pi) d S_{\mathrm{BH}}+\Phi d Q, \Phi$ being the electromagnetic scalar potential at the horizon. Thus

$$
\begin{aligned}
\left|\beta_{\omega \omega^{\prime}}\right|^{2} & =\exp \left[S_{\mathrm{BH}}(M-\omega, Q-e)-S_{\mathrm{BH}}(M, Q)\right] \\
& =\exp \left(-\Delta S_{\mathrm{BH}}\right), \quad\left|\alpha_{\omega \omega^{\prime}}\right|=1 .
\end{aligned}
$$

The density function, in the high energy approximation is [8]

$$
\rho\left(\frac{\omega-e}{T_{H}}\right) \approx \frac{\left|\beta_{\omega \omega^{\prime}}\right|^{2}}{\left|\alpha_{\omega \omega^{\prime}}\right|^{2}} .
$$

Therefore,

$$
\rho\left(\frac{\omega-e}{T_{H}}\right)=\exp \left(-\Delta S_{\mathrm{BH}}\right),
$$

and from Eq. (54), it is seen that this equals the value of $\rho\left(\omega+e / 2 T_{L}\right) \rho\left(\omega-e / 2 T_{R}\right)$ obtained from the $D$-brane picture.

The greybody factor $\sigma_{\text {abs }}$ for high energies is determined using the same methods as in the neutral emission case. The scalar equation is

$$
\begin{aligned}
(1+ & \left.\frac{r_{1}^{2}}{r^{2}}\right)\left(1+\frac{r_{5}^{2}}{r^{2}}\right)\left[\omega^{2}-e^{2}+(\omega \sinh \sigma-e \cosh \sigma)^{2} \frac{r_{0}^{2}}{r^{2}}\right] R \\
+ & \frac{h}{r^{3}} \frac{d}{d r}\left(h r^{3} \frac{d R}{d r}\right)=0 .
\end{aligned}
$$

By making the following transformations as in [10]:

$$
\omega^{\prime 2}=\omega^{2}-e^{2}, \quad e^{ \pm \sigma^{\prime}}=e^{ \pm \sigma}\left(\frac{\omega \mp e}{\omega^{\prime}}\right),
$$

$$
r_{n}^{\prime}=r_{0} \sinh \sigma^{\prime},
$$

we obtain Eq. (18) with $\omega \rightarrow \omega^{\prime}$ and $r_{n} \rightarrow r_{n}^{\prime}$. To order $\left(r_{0} / r_{1}\right)^{2}$, we again obtain the $\sigma_{\text {abs }}$ as in [10] as

$$
\sigma_{\mathrm{abs}}=g_{\mathrm{eff}} \omega^{\prime} \frac{e^{(\omega-e \Phi) / T_{H}}-1}{\left(e^{(\omega+e) / 2 T_{L}}-1\right)\left(e^{(\omega-e) / 2 T_{R}}-1\right)} .
$$

Here $(\omega+e) / T_{L}+(\omega-e) / T_{R}=(\omega-e \Phi) / T_{H}$ and $\Phi$ $=\tanh \sigma$ is the value of the scalar potential at the horizon. Thus clearly as $\omega^{\prime} / T_{L, R}>1, \sigma_{\mathrm{abs}} \rightarrow g_{\mathrm{eff}} \omega^{\prime}$. Inserting this value in Eq. (56), it is seen to match with Eq. (55).

\section{SCALAR EMISSION IN FOUR DIMENSIONS}

In this section, we briefly comment on the extension of our results to include high energy emission of scalar particles in the more realistic case of four dimensions. In the five dimensional case that we have analyzed, inclusion of the high energy effects did not affect the matching of the black hole and $D$-brane decay rates, even when the low energy condition of [10] was relaxed, up to leading order of the perturbation parameter that we considered. However, in this case we shall show that the same is not true, and that there is indeed a leading order correction to $\sigma_{\text {abs }}$ which means that the decay rates no longer match exactly as one goes beyond the low energy condition $\omega r_{1} \ll 1$. We shall not indicate the calculations explicitly, which are essentially in the same lines as in Sec. III, but rather state the main results. The results for charged scalar emission rates may be obtained by a simple extension [15]. The relevant wave equation whose solution we seek is $[15,16]$

$$
\frac{h}{r^{2}} \frac{d}{d r}\left(h r^{2} \frac{d R}{d r}\right)+\omega^{2} f R=0,
$$

where $f=\Pi_{i=1}^{4}\left(1+r_{i} / r\right), r_{i}$ 's being the parameters of the four-dimensional black hole. This equation can be expanded in powers of $1 / r$ in the near and far regions exactly as we did in the five-dimensional case. For the near region, keeping the next to leading order term in $1 / r$ leads to the equation

$$
\frac{d^{2} \psi}{d r^{2}}+\left(1+\frac{A}{r}\right) \omega^{2} \psi=0,
$$

where

$$
A=\left(r_{1}+r_{2}+r_{3}\right), \quad \psi=r R
$$


Interestingly, this equation is formally similar to the Coulomb wave equation. It has a solution in terms of confluent hypergeometric equations: namely,

$$
\psi=\alpha F(\eta \rho)+\beta G(\eta \rho),
$$

where $\rho=\omega r$ and $\eta=(-A \omega) / 2$ is a small parameter. The asymptotic form for this expression for large $r$ is given by

$$
F=g \cos \theta+f \sin \theta, \quad G=f \cos \theta-g \sin \theta,
$$

where $f$ and $g$ are constants depending on the black hole parameters: namely, to first order in $\eta$,

$$
\begin{gathered}
f=1+\frac{\eta}{2 \rho}, \\
g=-\frac{\eta}{4 \rho^{2}}, \\
\theta=\rho-\eta \ln 2 \rho-\eta \gamma,
\end{gathered}
$$

$\gamma$ being Euler's constant. The flux of incoming particles calculated from this form for the wave function at infinity is given by

$$
f_{\text {in }}=\frac{|\alpha|^{2}}{4}\left(-1-\frac{\eta}{2 \omega r}-\frac{\eta}{4 \omega^{2} r^{2}}\right)\left(-\omega+\frac{\eta}{r}\right) .
$$

It can be seen that most of the terms in the above expression can be neglected as $r \rightarrow \infty$ and we are left with

$$
f_{\text {in }}=\frac{|\alpha|^{2}}{4} \omega \text {. }
$$

Note that we have dropped a $\beta$-dependent piece in $f_{\text {in }}$ as exactly in the five-dimensional calculation it turns out to be extremely small compared to the $\alpha$-dependent term.

In the near region, Eq. (70) reduces after keeping the leading order terms in descending powers of $1 / r$ to

$$
\frac{h}{r^{2}} \frac{d}{d r}\left(h r^{2} \frac{d R}{d r}\right)+\left(\frac{A}{r^{4}}+\frac{B}{r^{3}}+\frac{C}{r^{2}}\right) \omega^{2} R=0 .
$$

In terms of the variables $a$ and $b$ defined according to

$$
(a+b)^{2}=4(A+B+C) ; a b=B+C .
$$

very close to the horizon we can once again make the substitution $y=-\ln (1-v)$ so that Eq. (77) reduces to the simple harmonic equation

$$
\frac{d^{2} R}{d y^{2}}+(a+b) R=0
$$

and, just as in the five-dimensional case, $a$ and $b$ are related to the black hole parameters, the relation being given by

$$
\begin{aligned}
& a=\frac{\omega}{4 \pi T_{R}}+2 \pi \omega T_{H}\left(r_{1} r_{3}+r_{2} r_{3}+r_{1} r_{2}\right), \\
& b=\frac{\omega}{4 \pi T_{L}}+2 \pi \omega T_{H}\left(r_{1} r_{3}+r_{2} r_{3}+r_{1} r_{2}\right) .
\end{aligned}
$$

Again writing the incoming solution as

$$
R=K \exp [-i \sqrt{A+B+C} \ln (1-v)]
$$

the incoming flux at the black hole horizon is calculated to be

$$
f_{\mathrm{abs}}=|K|^{2} r_{0} \frac{(a+b)}{2} \text {. }
$$

Now, matching the near and the far zone solutions as in [10] gives the relation

$$
\frac{K}{\alpha}=\frac{\omega}{E}\left(1-\frac{\pi \eta}{2}\right)
$$

where $E$ is given in terms of $\Gamma$ functions as before as

$$
E=\frac{\Gamma(1-i a-i b)}{\Gamma(1-i a) \Gamma(1-i b)},
$$

so that the calculation for $\sigma_{\text {abs }}$ finally yields

$$
\sigma_{\mathrm{abs}}=\frac{4 \pi}{\omega^{2}} \frac{f_{\mathrm{abs}}}{f_{\mathrm{in}}}=\sigma_{\mathrm{abs}}^{0}[1-\pi \eta]
$$

where $\sigma_{\mathrm{abs}}^{0}=4 \pi^{2} r_{1} r_{2} r_{3} \omega$ at high energies. Hence we see from Eq. (84) that there is an order $\eta$ correction to $\sigma_{\text {abs }}$, in contrast to the five-dimensional case where this correction was negligible. This, along with the microcanonical condition implies that the $D$-brane and black hole decay rates match only in the energy regime given by

$$
\sqrt{\frac{r_{0}}{r_{1}}} \ll \omega r_{1} \ll 1,
$$

where the emission rates in four dimensions is given by

$$
\Gamma_{H}=\Gamma_{D}=4 \pi^{2} r_{1} r_{2} r_{3} \omega \exp \left(-\Delta S_{\mathrm{BH}}\right) \frac{d^{3} k}{(2 \pi)^{3}} .
$$

The difference between the five- and four-dimensional cases that we have dealt with is also apparent from the general analysis of [16]. The far zone equation that was effectively the source of the difference in the two different dimensions can be written to leading order for $D$ dimensions as

$$
\frac{d^{2} \psi}{d \rho^{2}}+\left[1-\frac{(D-2)(D-4)}{\rho^{2}}\right] \psi=0,
$$

where $\rho=\omega r$, and $R=r^{-(D-2) / 2} \psi$. The general solution of the above equation is

$$
F=\sqrt{\frac{\pi}{2}} \rho^{1 / 2} J_{(D-3) / 2}(\rho) .
$$

In five dimensions, the addition of an interaction term also of the form $1 / \rho^{2}$ simply modifies the order of the Bessel function. The resulting corrections in $\sigma_{\text {abs }}$ is negligible. However, in four dimensions, there is a new $1 / \rho$ term, which gives rise to the Coulomb wave function and a leading order correction in the final result. 


\section{DISCUSSIONS}

In this paper, we have compared black hole and $D$-brane decay rates for neutral and charged scalar emission at high energies. The microcanonical picture was used in the $D$-brane side, which naturally incorporates the condition $\omega / T_{L, R} \gg 1$. This condition was crucially used in calculating the black hole greybody factor. In five dimensions, the decay rates match for all values of energy consistent with the microcanonical picture, while in four dimensions, they match in a restricted range. It would be interesting to investigate this difference and see if a more careful analysis can bring about an exact matching in four dimensions.

In our calculation of high energy greybody factors, it was assumed that there were no explicit back reaction effects as in the case of the Hawking spectrum. This can be justified as follows: the modified black hole metric due to back reaction of the shell can be approximated by the original metric with a shift in the Arnowitt-Deser-Misner (ADM) mass of the black hole by $\omega$. The corresponding effect on the $D$-brane is the reduction of the excitation energy of the gas of open strings, i.e., $E=E_{L}+E_{R} \rightarrow E-\omega$. Since $E$ can be written as

$$
E=\frac{\pi r_{0}^{2} \cosh 2 \sigma}{8 G_{5}}
$$

the parameters $r_{0}$ and $\sigma$ are changed accordingly. It can be shown that these changes result in a correction also of order $\left(r_{0} / r_{1}\right)^{2}$ in $\sigma_{\text {abs }}$ and hence can be ignored.

\section{ACKNOWLEDGMENTS}

We thank S. R. Das, T. Jayaraman, P. Majumdar, P. Ramadevi, and G. Sengupta for discussions. One of us (A.D) would like to thank G. Date for discussions. We thank the organizers of the Puri Workshop, for hospitality, where part of the work was done.
[1] A. Sen, Mod. Phys. Lett. A 10, 2081 (1995).

[2] A. Strominger and C. Vafa, Phys. Lett. B 379, 99 (1996).

[3] J. M. Maldacena and A. Strominger, Phys. Rev. Lett. 77, 428 (1996); C. V. Johnson, R. R. Khuri, and R. C. Myers, Phys. Lett. B 378, 78 (1996); M. Cvetic and D. Youm, Phys. Rev. D 54, 2612 (1996); G. Horowitz, Report No. gr-qc/9604051 (unpublished); A. A. Tseytlin, Nucl. Phys. B477, 431 (1996); N. Hambli, Phys. Rev. D 54, 5129 (1996).

[4] C. Callan and J. Maldacena, Nucl. Phys. B475, 645 (1996).

[5] J. Maldacena and L. Susskind, Nucl. Phys. B475, 679 (1996).

[6] S. R. Das and S. D. Mathur, Nucl. Phys. B478, 561 (1996).

[7] S. R. Das and S. D. Mathur, Phys. Lett. B 375, 103 (1996).

[8] E. Keski-Vakkuri and P. Kraus, Report No. hep-th/9610045 (unpublished).

[9] W. G. Unruh, Phys. Rev. D 14, 3251 (1976).

[10] J. Maldacena and A. Strominger, Phys. Rev. D 55, 861 (1997).

[11] S. R. Das, G. Gibbons, and S. D. Mathur, Phys. Rev. Lett. 78, 417 (1997).

[12] G. Horowitz, J. Maldacena, and A. Strominger, Phys. Lett. B 383, 151 (1996).

[13] S. S. Gubser and I. R. Klebanov, Nucl. Phys. B482, 173 (1996).

[14] P. Kraus and F. Wilczek, Nucl. Phys. B433, 665 (1994); B437, 231 (1994).

[15] S. S. Gubser and I. R. Klebanov, Phys. Rev. Lett. 77, 4491 (1996).

[16] S. P. de Alwis and K. Sato, Phys. Rev. D 55, 6181 (1997). 